lich. Das rezidivfreie und das Gesamtüberleben waren ebenfalls in beiden Studienarmen identisch. Letztendlich ergänzt diese Studie auch die ACOSOG-Z0011-Daten, dass eine axilläre Chirurgie bei befallenem SentinelLymphknoten nicht zwingend notwendig ist.

5. Die Daten der ATTOM-Studie wurden von Richard Gray und Kollegen präsentiert (\#5). 6.953 Hormonrezeptor-positive Patientinnen wurden randomisiert in fünf versus zehn Jahre Tamoxifen. Die Studie bestätigte die Daten der ATLAS-Studie (mit
11.646 Patientinnen). Die relative Risikoreduktion von Rezidiven und Metastasen betrug durch die zusätzlichen fünf Jahre Tamoxifen $15 \%$. Das Endometriumkarzinomrisiko betrug 2,9\% im 10-Jahres-Arm verglichen mit 1,3\% im 5-Jahres-Arm. Das relative Mortalitätsrisiko wurde um $6 \%$ verringert. Die Brustkrebs-bedingte Mortalität wurde in beiden Studien (ATTOM und ATLAS) um 3\% absolut gesenkt. Beide Studien haben zu einer Änderung der therapeutischen Leitlinien geführt, beantworten jedoch nicht die wichtige Frage, wel- ches Patientinnenkollektiv speziell von einer solchen Therapie profitiert und für welches Patientinnenkollektiv unter Umständen die Nebenwirkungen größer sind als die Wirksamkeit dieser verlängerten adjuvanten Therapie.

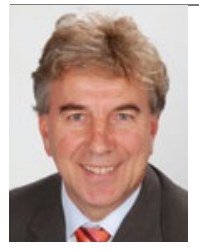

Prof. Dr. med. Michael Untch Klinik für Gynäkologie und Geburtshilfe, HELIOS Klinikum Berlin-Buch

\title{
Maligne Hauttumoren: Immunologische Checkpoint-Kontrolle
}

Der diesjährige ASCO in Chicago bescherte abermals deutlich sichtbare Fortschritte in der Behandlung von malignen Hauttumoren. Besonderer Höhepunkt war das Symposium zur immunologischen Checkpoint-Kontrolle blockierender Antikörper. Hier zeigten mehrere Antikörper sowohl gegen PD-1 als auch gegen PD-1L, dass viele Melanompatienten sehr gut und sehr lang andauernd - z. T. bis über zwei Jahre auf diese Therapie ansprachen. Darüber hinaus wurden erste Kombinationsdaten mit dem anti-CTLA4-Antikörper Ipilimumab sowie einem PD-1-Antikörper gezeigt, deren klinisches Ansprechen sehr umfangreich und voll- ständig war. Derzeit befinden sich sowohl verschiedene PD-1-Antikörper als auch deren Kombination in der klinischen Phase-III-Erprobung - auch in deutschen Zentren.

Zweiter Höhepunkt war die Weiterentwicklung zielgerichteter Therapien beim Melanom. Die Kombination von BRAF- und MEK-Inhibitoren scheint nicht nur das klinische Ansprechen, sondern auch die Überlebenszeit von Melanompatienten mit BRAF-Mutation noch einmal deutlich zu verlängern im Vergleich zu Vemurafenib alleine. Derzeit werden in Deutschland auch für zwei Zulassungsstudien Patienten rekrutiert.
Darüber hinaus hat die zielgerichtete Therapie einen weiteren Hauttumor, das fortgeschrittene Basalzellkarzinom, erreicht. Hier wurde gezeigt, dass mit dem Hedgehog-Inhibitor Vismogenib ein deutliches Ansprechen erzielt werden kann. Diese Daten werden im Laufe des Sommers zur Zulassung auch in Deutschland führen.

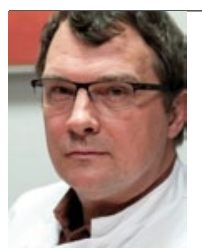

Prof. Dr. med.

Dirk Schadendorf Klinik für Dermatologie Universitätsklinikum Essen

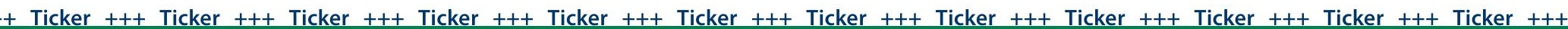

DLBCL: routinemäßig kein Kontroll-CT in der Nachsorge

Eine routinemäßige Computertomografie (CT)-Kontrolle in der Nachsorge von Patienten mit diffusem großzelligem B-Zelllymphom (DLBCL) hat nur begrenzten Nutzen für den Patienten. Die ist das Ergebnis einer Studie der Arbeitsgruppe um Carrie A. Thomssen [J Clin Oncol. 2013;31(suppl;abstr. 8504)]. In der multizentrischen Studie waren 644 Patienten mit DLBCL, die in der First-Line-Therapie mit Anthrazyklin-basierter Immuno- chemotherapie behandelt worden waren, von 2002 bis 2009 nachbeobachtet. $42 \%$ der Patienten erlitten innerhalb eines Jahres nach Diagnose einen Rückfall. $27 \%$ innerhalb von zwei Jahren und $31 \%$ nach drei und mehr Jahren. Bei nur $8 \%$ der Patienten wurde die Progression der Erkrankung durch ein CT entdeckt; in der Mehrzahl der Fälle waren es klinische Symptome, die erste Hinweise lieferten. Nur bei zwölf Patienten wurden die Veränderungen während des geplanten Nachsorgetermins festgestellt. (db)
Inhibition von PI3K-delta: eine Option bei CLL?

In einer Phase-III-Studie wird derzeit untersucht, ob Patienten mit chronischer lymphatischer Leukämie (CLL), die sich einer Behandlung mit Bendamustin plus Rituximab unterziehen, von der zusätzlichen Inhibition der Phosphoinositide-3-Kinase (PI3K)-delta profitieren [Eradat $\mathrm{HA}$ et al. J Clin Oncol. 2013;31(suppl;abstr. TPS7133)]. In die multizentrische Studie sollen insgesamt 390 CLL-Patienten eingeschlossen werden, die in- nerhalb von 36 Monaten nach Abschluss einer Erstlinientherapie einen Rückfall erlebt haben. Alle Patienten erhalten 6 Zyklen Rituximab (Zyklus 1: $375 \mathrm{mg} / \mathrm{m}^{2}$ an Tag 1 und Zyklus 2-5: 500 $\mathrm{mg} / \mathrm{m}^{2}$ an Tag 1; q4w) plus $6 \mathrm{Zy}$ klen Bendamustin $\left(70 \mathrm{mg} / \mathrm{m}^{2}\right.$ an Tag 1 und 2; q4w) sowie im Verhältnis 1:1 randomisiert zusätzlich den oralen PI3K-delta-Inhibitor Idelalisib in einer Dosierung von $150 \mathrm{mg}$ BID oder Placebo. Als primären Endpunkt haben die Wissenschaftler das progressionsfreie Überleben gewählt. Sekundäre Endpunkte 